\title{
Enfoque na familia sobre tuberculose sob a ótica dos agentes comunitários de saúde
}

\author{
I ${ }^{1}$ Rebeca Sousa Braga, ${ }^{2}$ Tatiane Cabral Siqueira, ${ }^{3}$ Valeria Moreira da Silva, \\ ${ }^{4}$ Nathalia Halax Orfão I
}

Resumo: Para o controle da tuberculose (TB), é imprescindível a atuação dos agentes comunitários de saúde (ACS) desde o diagnóstico até o desfecho dos casos. Este estudo teve como objetivo analisar a dimensão enfoque na família sobre a TB, sob a ótica dos ACS em Porto Velho-RO. Trata-se de estudo descritivo, do tipo inquérito, realizado de forma transversal a partir de abordagem quantitativa, com os ACS que atuavam na APS da zona urbana do município por meio de entrevistas com o questionário Primary Care Assessment TooI (PCATool), validado para o Brasil e adaptado para a atenção à TB. Os dados foram analisados a partir da estatística descritiva, após atender os preceitos éticos. Foram entrevistados 119 ACS, que sempre questionavam as condiçóes de vida, tinham conhecimento sobre as pessoas que moravam com o doente de TB e/ou família, solicitavam informaçôes sobre as enfermidades, questionavam a apresentação dos sintomas da doença, solicitavam exame de escarro e/ou raio $\mathrm{X}$ e/ou PPD e orientavam sobre a doença, tratamento e outros problemas de saúde. Tais achados suscitam reflexóes sobre a fragilidade dos princípios da longitudinalidade e integralidade para maior resolutividade da APS como ordenadora da RAS e coordenadora do cuidado.

> Palavras-chave: tuberculose; agentes comunitários de saúde; Atenção Primária à Saúde; pesquisa sobre serviços de saúde.

\author{
${ }^{1}$ Fundação Universidade Federal \\ de Rondônia (UNIR). Porto Velho- \\ RO, Brasil (rebeca.braga.rs@ \\ gmail.com). \\ ORCID: 0000-0001-6046-3670 \\ 2 Fundação Universidade Federal \\ de Rondônia (UNIR), Porto Velho- \\ RO, Brasil (tatianecabralsiqueira@ \\ gmail.com). \\ ORCID: 0000-0002-3860-1261 \\ ${ }^{3}$ Departamento de Enfermagem, \\ Fundação Universidade Federal de \\ Rondônia (UNIR), Porto Velho-RO, \\ Brasil (valeria.moreira@unir.br). \\ ORCID: 0000-0001-6786-6325 \\ ${ }^{4}$ Departamento de Medicina, \\ Fundação Universidade Federal de \\ Rondônia (UNIR), Porto Velho-RO, \\ Brasil (nathaliahalax@unir.br). \\ ORCID: 0000-0002-8734-3393
}

Recebido em: 13/05/2020 Aprovado em: 17/11/2020 Revisado em: 29/03/2021 


\section{Introdução}

A tuberculose (TB) é uma das 10 principais causas de morte no mundo. Em 2018, a doença acometeu cerca de 10 milhóes de pessoas e ocasionou 1,451 milhão de óbitos (WHO, 2019). No Brasil, foram notificados 75.239 casos novos e 4.490 óbitos, o que equivale ao coeficiente de incidência e mortalidade de 34,8 casos e 2,2 óbitos/100 mil hab., respectivamente (BRASIL, 2020). No mesmo ano, Rondônia apresentou 533 casos novos de TB, sendo a maioria diagnosticada em Porto Velho (353 casos - 66,2\%), o qual também apresenta elevado coeficiente de incidência (66,7 casos /100 mil hab.) em comparação ao estado (28,5 casos/ 100 mil hab.), bem como baixo percentual de cobertura do tratamento diretamente observado (TDO) (2,7\% dos casos de TB pulmonar) e a segunda capital em percentual de abandono do tratamento da TB (24,6\%) no país (BRASIL, 2019; BRASIL, 2020).

Tais dados epidemiológicos representam a importância da atuação da Atenção Primária à Saúde (APS) como porta de entrada preferencial ao serviço de saúde atuando na prevenção e proteção à saúde, desde o rastreamento dos sintomáticos respiratórios (SR) para o diagnóstico precoce até o encerramento dos casos, incluindo estratégias que possibilitem a adesão e sucesso da terapêutica, tais como o TDO e a inserção dos agentes comunitários de saúde (ACS) na Estratégia Saúde da Família (ESF).

Essa inserção contribui na realização da busca ativa de SR por meio de visitas domiciliares (VD) para elucidação diagnóstica o mais precoce possível, além de auxiliar no acompanhamento dos casos em tratamento, orientaçóes sobre a doença, tratamento, efeitos colaterais, dentre outros, além da supervisão da ingesta medicamentosa através do TDO. Tal perspectiva potencializa, ainda, o fortalecimento do vínculo entre o serviço de saúde, profissionais, indivíduo, família e comunidade, sendo este o principal subsídio de atuação dos ACS (FERREIRA et al., 2019; GONZALES et al., 2015; PEREIRA et al., 2017).

Furlan, Gonzales e Marcon (2015) reforçam que apenas a descentralização do tratamento da TB para a APS não se constitui de forma isolada na melhoria nos resultados dos tratamentos dos doentes de $\mathrm{TB}$, mas exige a inserção da família, a qual deve ser incluída pelos profissionais de saúde desde as açôes de vigilância e controle da doença, com o oferecimento de suporte e orientaçôes, tendo em vista que estas são a fonte de cuidado e apoio. Neste sentido, este estudo teve como objetivo analisar a dimensão enfoque na família sobre a TB, sob a ótica dos ACS em Porto Velho-RO. 


\section{Metodologia}

Trata-se de estudo descritivo, do tipo inquérito, realizado de forma transversal a partir de abordagem quantitativa realizado em Porto Velho, capital do estado de Rondônia, que atualmente possui 17 Unidades Básicas de Saúde (UBS) e 37 Unidades de Saúde da Família (USF), totalizando 54 unidades da APS. Estas se distribuem na zona urbana (20) e rural (33), subdivididas em unidades terrestres (18) e ribeirinhas (15), bem como uma unidade móvel fluvial (BRASIL, 2018). Neste estudo, foram considerados somente os estabelecimentos de saúde distribuídos na zona urbana.

A atenção à TB no município é descentralizada para APS, a qual é responsável por realizar açôes para identificar os SR, solicitar exames diagnósticos, oferecer o teste HIV, notificar os casos no Sistema de Informação de Agravos de Notificação (SINAN), acompanhar os casos de TB em tratamento, identificando os faltosos, oferecer o Tratamento Diretamente Observado (TDO), investigar os contatos e encaminhar para outros serviços, se necessário.

Como rede de apoio e suporte, no nível ambulatorial, o município conta com um Centro de Especialidades Médicas (CEM) para os casos de TB extrapulmonar e TB infantil, além de um Serviço de Atendimento Especializado (SAE) para aqueles que possuem coinfecção TB/HIV. Para casos de TB droga resistente (TBDR) e aqueles que necessitem de internação, o estado possui um hospital como referência a nível terciário.

A população do estudo foi constituída pelos ACS que atuam na APS da zona urbana de Porto Velho, a partir da partilha proporcional realizada no projeto intitulado "Dimensóes organizacionais e de desempenho dos serviços de saúde para o manejo do cuidado aos doentes de tuberculose em Porto Velho-RO", do qual este estudo faz parte. Foram considerados critérios de inclusão aqueles que exerciam sua função por pelo menos 12 meses e que tinham lidado com pelo menos um caso de TB desde sua formação. E como exclusão, todos aqueles que estavam de férias e/ou licença durante o período de coleta de dados. Tendo em vista a população de 737 profissionais nessas unidades (CNES, 2018), o $n$ amostral foi estimado pela fórmula de cálculo amostral com população finita:

$$
n=\frac{z_{\frac{\alpha}{2}}^{2} * N * P *(1-P)}{\varepsilon^{2} *(N-1)+z_{\frac{\alpha}{2}}^{2} *(1-P)}
$$


Considerando um erro $(\varepsilon)$ de $5 \%$, um intervalo confiança $(Z)$ de $95 \%$, proporção amostral $(\mathrm{P})$ de $50 \%$ da população $(\mathrm{N})$, deveriam ser entrevistados, no mínimo, 253 profissionais, dos quais 136 deveriam ser ACS das diferentes unidades da APS da área urbana do município, sem estratificação desses profissionais por unidade. A abordagem ocorreu de forma aleatória na unidade, após contato para explicar os objetivos do estudo e confirmar o quantitativo de profissionais com a direção, onde também se realizou a coleta de dados em local que resguardava a privacidade dos participantes.

Os dados foram coletados, no período de maio de 2018 a fevereiro de 2019, por seis alunos de graduação e uma enfermeira nas unidades de saúde após treinamento prévio com a coordenadora responsável pelo projeto matriz. Foi utilizado o questionário Primary Care Assessment TooI (PCATool) (STARFIELD, 2002), validado para o Brasil (ALMEIDA; MACINKO, 2006) e adaptado para a atenção à TB (VILLA; RUFFINO-NETTO, 2009). Para atender o objetivo deste estudo, considerou-se apenas a dimensão enfoque na família da versão para profissionais de saúde, a qual possui oito variáveis (quadro 1), e possui como categorias de respostas segundo a escala de Likert, com os valores entre 1 a 5 , referente ao grau de relação de preferência ou concordância com as afirmaçôes.

Quadro 1. Variáveis relacionadas à dimensão enfoque na família selecionada para este estudo

\begin{tabular}{|c|c|}
\hline Dimensão & Variáveis \\
\hline \multirow{8}{*}{$\begin{array}{l}\text { Enfoque } \\
\text { na família }\end{array}$} & $\begin{array}{l}\text { Questionamento sobre as condiçóes de vida das pessoas que moravam com o doente de TB e/ou da } \\
\text { sua família (emprego, moradia, saneamento básico), durante as consultas, pelos profissionais de saúde. }\end{array}$ \\
\hline & $\begin{array}{l}\text { Conhecimento pelos profissionais de saúde sobre as pessoas que moravam com o doente de TB e/ } \\
\text { ou família. }\end{array}$ \\
\hline & $\begin{array}{l}\text { Solicitação de informaçóes pelos profissionais de saúde sobre as enfermidades das pessoas que } \\
\text { moravam com o doente de TB e/ou família. }\end{array}$ \\
\hline & $\begin{array}{l}\text { Questionamento pelos profissionais de saúde sobre a apresentação dos sintomas da doença das } \\
\text { pessoas que moravam com o doente de TB e/ou família. }\end{array}$ \\
\hline & $\begin{array}{l}\text { Solicitação pelos profissionais de saúde de exame de escarro e/ou raio X e/ou PPD para as pessoas } \\
\text { que moravam com o doente de TB e/ou família. }\end{array}$ \\
\hline & $\begin{array}{l}\text { Orientação pelos profissionais de saúde sobre a doença com as pessoas que moravam com o doente } \\
\text { de TB e/ou família. }\end{array}$ \\
\hline & $\begin{array}{l}\text { Orientação pelos profissionais de saúde sobre o tratamento com as pessoas que moravam com o } \\
\text { doente de TB e/ou família. }\end{array}$ \\
\hline & $\begin{array}{l}\text { Orientação pelos profissionais de saúde sobre outros problemas de saúde com as pessoas que } \\
\text { moravam com o doente de TB e/ou família. }\end{array}$ \\
\hline
\end{tabular}

Fonte: Villa e Ruffino-Netto (2009). 
Os dados coletados foram armazenados no software Microsoft Excel e analisados por meio de estatística descritiva no software Statistica 13.4, da TIBCO. Determinouse um escore médio para cada variável que corresponde à somatória de todas as categorias de respostas dividido pelo total de participantes. De modo complementar, definiu-se um escore médio da dimensão elencada para este estudo, que corresponde à somatória de todos os escores médios das variáveis dividido pelo total de variáveis (VILLA; RUFFINO-NETTO, 2009). Posteriormente, conforme a escala Likert de resposta do instrumento, adotaram-se os seguintes parâmetros para categorizar os escores médios obtidos, a saber: nunca ( 0 a 1$)$, quase nunca ( 1,01 a 2$)$, às vezes $(2,01$ a 3), quase sempre (3,01 a 4) e sempre (4,01 a 5).

Atendendo às recomendaçóes da Resolução n ${ }^{\circ} 466 / 12$ do Conselho Nacional de Saúde (BRASIL, 2012), o projeto matriz, intitulado "Dimensóes organizacionais e de desempenho dos serviços de saúde para o manejo do cuidado aos doentes de tuberculose em Porto Velho-RO” foi aprovado pelo Comitê de Ética em Pesquisa da Universidade Federal de Rondônia, conforme o parecer n ${ }^{\circ}$.585.934. As entrevistas ocorreram após assinatura do Termo de Consentimento Livre e Esclarecido (TCLE) pelos ACS, na unidade de saúde em que eles atuavam e local que resguardassem a privacidade dos mesmos.

\section{Resultados}

Ainda que 397 ACS estivessem cadastrados no CNES, verificou-se que apenas 362 eram atuantes nas unidades de saúde do município. Destes, 190 foram abordados durante a coleta de dados, mas 65 foram excluídos por não atenderem aos critérios previamente estabelecidos e seis por se recusarem a participar da pesquisa. Assim, 119 ACS foram entrevistados. Ressalta-se que 172 ACS foram excluídos por outros motivos, tais como não atuavam mais na unidade e/ou não foram encontrados mesmo após três tentativas.

Quanto à dimensão enfoque na família (média do escore geral = 4,74 - sempre), sob a ótica dos ACS, estes sempre questionavam sobre as condiçóes de vida das pessoas que moravam com o doente de TB e/ou da sua família; os profissionais tinham conhecimento sobre as pessoas que moravam com o doente de TB e/ou família, solicitavam informaçóes sobre as enfermidades das pessoas que moravam com o doente de TB e/ou família, questionavam a apresentação dos sintomas da 
doença sobre as pessoas que moravam com o doente de TB e/ou família, solicitavam exame de escarro e/ou raio X e/ou PPD para as pessoas que moravam com o doente de TB e/ou família, e orientavam sobre a doença, tratamento e outros problemas de saúde com as pessoas que moravam com o doente de TB e/ou família (Gráfico 1).

Gráfico 1. Representação gráfica da média e do intervalo de confiança dos escores da dimensão enfoque na família, obtidos pelos ACS em Porto Velho-RO, maio/2018 a fevereiro/2019

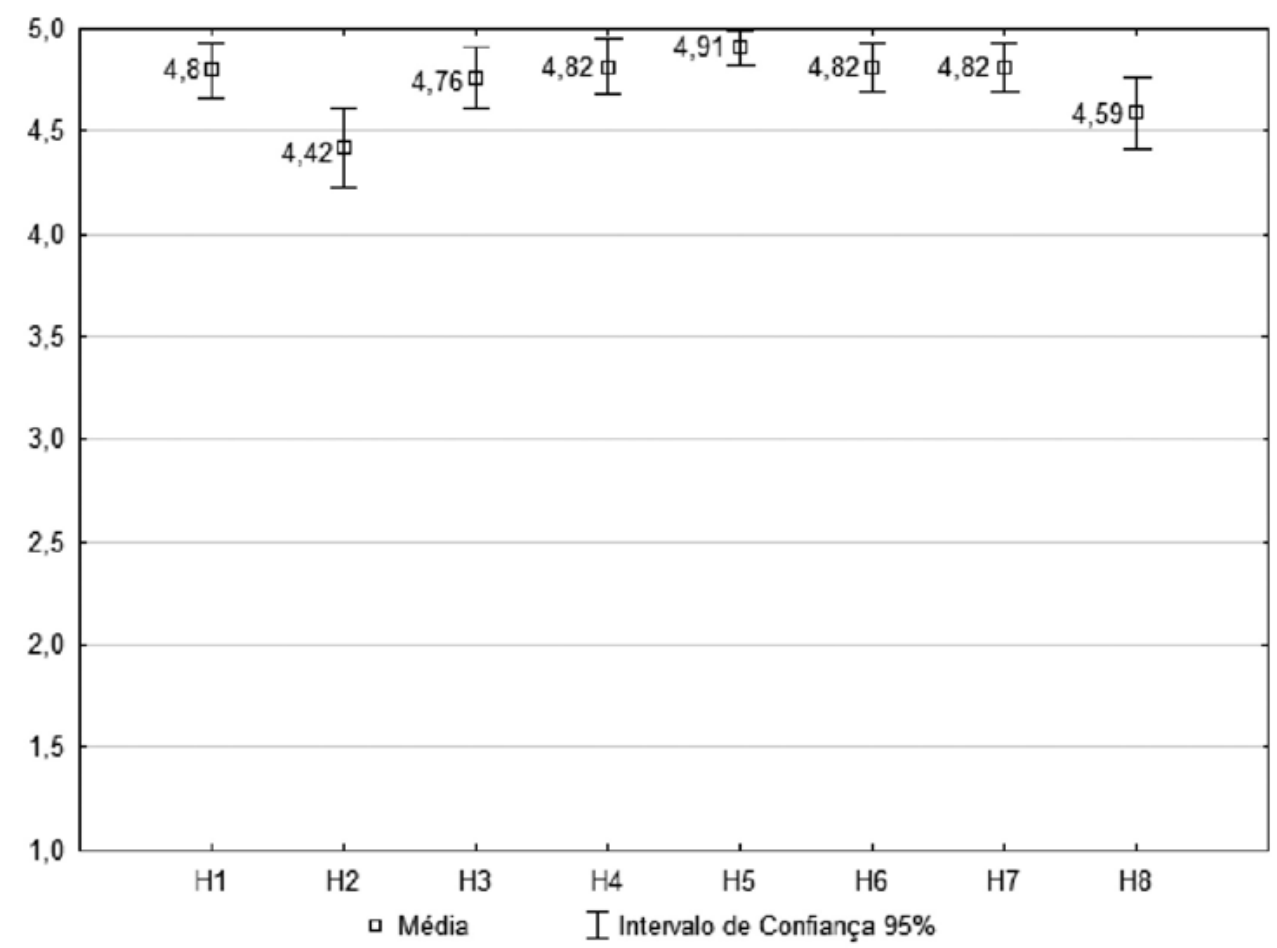

Legenda - H1 - Questionamento sobre as condições de vida das pessoas que moravam com o doente de TB e/ou da sua família H2- Conhecimento pelos profissionais de saúde sobre as pessoas que moravam com o doente de TB e/ou família $\mathbf{H 3}$ - Solicitação de informações pelos profissionais de saúde sobre as enfermidades das pessoas que moravam com o doente de TB e/ou família. H4 - Questionamento pelos profissionais de saúde sobre a apresentaçâo dos sintomas da doença das pessoas que moravam com o doente de TB e/ou família. H5 - Solicitação pelos profissionais de saúde de exame de escarro e/ou raio $\mathrm{X}$ e/ou PPD para as pessoas que moravam com o doente de TB e/ou família. H6 - Orientaçấo pelos profissionais de saúde sobre a doença com as pessoas que moravam com o doente de TB e/ou família. H7 - Orientação pelos profissionais de saúde sobre o tratamento com as pessoas que moravam com o doente de TB e/ou família. H8 - Orientação pelos profissionais de saúde sobre outros problemas de saúde com as pessoas que moravam com o doente de TB e/ou família. 


\section{Discussão}

Os resultados obtidos neste estudo mostram que, sob a ótica dos ACS, o enfoque na família ocorre sempre na APS, ainda que contraditório aos dados epidemiológicos, cujo coeficiente de incidência no município é maior quando comparado com o estado e país. Este é reflexo da continuidade da cadeia de transmissão, além de desfechos desfavoráveis com a interrupçáo do tratamento que poderiam ser modificados considerando a inserção da família e comunidade em que o indivíduo está inserido. De modo complementar, verificou-se durante as entrevistas, a partir das falas dos participantes, que o manejo do cuidado da TB está centralizado nos enfermeiros e médicos, sem que ocorra a participação e discussão dos casos entre os membros da equipe. Além disso, estudos ressaltam que tanto a busca ativa de SR, quanto o tratamento, na maioria das vezes ocorrem sem a investigação dos contatos, indicando fragilidades nessa dimensão (PEREIRA et al., 2018; FURLAN et al., 2015).

No que concerne ao questionamento acerca das condiçóes de vida e conhecimento pelos profissionais de saúde sobre as pessoas que moravam com o doente de TB e/ ou família, estudos apontam que, apesar da necessidade do conhecimento sobre a realidade das famílias, nem sempre são questionados (PEREIRA et al., 2018; FURLAN et al., 2015). De modo complementar, apenas questionar não significa conhecer; é essencial o estreitamento do vínculo por meio da realização da VD, possibilitando a observação das subjetividades, bem como se caracteriza como uma das responsabilidades e estratégias que deveriam ser efetivadas pela APS.

A VD é uma ferramenta fundamental para abordagem à família, e do (re) conhecimento do contexto em que esta e o caso-índice estão inseridos, auxiliando na identificação dos contatos domiciliares, diagnóstico precoce, análise de possíveis causas de abandono ao tratamento. Além disso, oportuniza momentos de orientações no âmbito familiar para suporte e apoio, visando à continuidade do tratamento pelo doente de TB. No entanto, tal açâo não é realizada rotineiramente e, quando ocorre, é focada nos idosos e acamados com doenças crônicas não transmissíveis e de forma fragmentada, desarticulada com a TB, ainda que este grupo seja vulnerável (PEREIRA et al., 2017; PEREIRA et al., 2018).

A respeito da solicitação de informaçôes sobre as enfermidades e questionamento, pelos profissionais de saúde, sobre a apresentação dos sintomas da doença das pessoas que moravam com o doente de TB e/ou família, pode-se aferir o cuidado 
centralizado no caso-índice, que se distancia das ações de vigilância em saúde no que concerne aos aspectos preventivos, tais como a avaliação dos contatos e rastreamento dos SR (FURLAN et al., 2015).

Tais informaçóes poderiam subsidiar o diagnóstico precoce e iniciar a quimioprofilaxia, quando necessário. Além disso, para obter uma prática integral e resolutiva, énecessário associar outros problemas de saúde como fatores que aumentam a vulnerabilidade para o adoecimento por $\mathrm{TB}$, bem como ter conhecimento sobre a doença para a identificação precoce dos SR e incluir a família, tendo em vista os riscos de contaminação e cuidado ao doente de TB (GASPAR et al., 2019).

No que se refere à solicitação, pelos profissionais de saúde, de exame de escarro e/ou raio X e/ou PPD para as pessoas que moravam com o doente de TB e/ou família, segundo estudos, a falta de conhecimento sobre a doença é uma fragilidade que afeta a qualidade do serviço prestado, repercutindo na demora e dificuldade de identificação de casos suspeitos e manejo inadequado (GASPAR et al., 2019; ROCHA et al., 2015). A não solicitação de exames para os contatos reflete também nas fragilidades das práticas de vigilância em saúde para o controle da TB, que deve abranger não apenas o indivíduo, mas o contexto em que o mesmo se insere, considerando a importância da implementação de açóes efetivas de prevenção e rastreamento dos SR.

Wysocki et al. (2017) destacam, ainda, que a organização dos serviços em que as informações dos casos são centralizadas ao enfermeiro levam ao desconhecimento da disponibilidade de insumos nos serviços pelos ACS. Isso prejudica a realização da solicitação por esses profissionais, bem como a inclusão destes nas ações de controle da TB como integrantes da equipe e responsáveis pelos usuários.

No que se refere às orientaçóes sobre sintomas, tratamento e outros problemas de saúde, estudos apontam que ainda hoje o estigma da doença, seja por parte da comunidade ou dos profissionais, é um entrave para a continuidade do tratamento, ações de educação em saúde e até mesmo aceitação pela comunidade (LINHARES et al., 2020; GONZALES et al., 2015).

Rocha et al. (2015) apontam ainda sobre as lacunas no nível de conhecimento dos profissionais sobre biossegurança e identificação dos sinais e sintomas dos casos de TB, que repercutem em orientaçôes fragmentadas ao doente, família e comunidade. Tal fato, aliado à melhora dos sinais e sintomas após o início do tratamento, implicam em uma percepção errônea e equivocada de cura, 
que repercute no abandono ao tratamento, desfechos desfavoráveis dos casos e continuidade no ciclo de transmissão (GEBREWELD et al., 2018).

Como limitação deste estudo, ressalta-se a importância de incluir e contrapor a ótica dos doentes de TB e/ ou família sobre a dimensão analisada, considerando a diferença na concepção entre oferta e recebimento do serviço pelos profissionais e usuários, respectivamente.

\section{Considerações finais}

Assim, ainda que este estudo não tenha encontrado fragilidades na dimensão enfoque na família sob a ótica dos ACS, suscitam-se reflexóes a respeito dos fatores que contrapóem o resultado obtido, pois nem sempre há realização de VD. Muitas vezes a população acometida por TB parece se encontrar "fora de área” ou em "área descoberta”, denominaçôes que contrariam a Política Nacional da Atenção Básica, mas que em diversos momentos são relatadas e "justificadas" pelos profissionais de saúde.

É notório que a presença do ACS contribui na melhoria dos indicadores da ESF em comparação a UBS tradicional, considerando que este é o profissional mais próximo do doente, família e comunidade pelas próprias atribuiçôes definidas e desempenhadas. Entretanto, questiona-se: se forem somados esforços na formação, valorização e maior inclusão do ACS no processo de trabalho e cuidado, aonde podemos chegar?

Tais aspectos permitem refletir, ainda, sobre a fragilidade dos princípios da longitudinalidade e integralidade para maior resolutividade da APS como ordenadora da RAS e coordenadora do cuidado. Esta poderia se apropriar e fortalecer o vínculo para conhecer a realidade dos doentes de TB e família, sobre os quais possui responsabilidade sanitária, além de promover educação em saúde efetiva sobre a TB, tratamento e outras enfermidades, para garantir a adesão terapêutica, diminuindo o estigma e possíveis complicaçóes.

\section{Referências}

ALMEIDA, C.; MACINKO, J. Validação de uma metodologia de avaliação rápida das caracteristicas organizacionais e do desempenho dos serviços de atenção básica do Sistema Único de Saúde (SUS) em nivel local. Brasília: Organização Pan-Americana da Saúde/OPAS, 2006

BRASIL. Ministério da Saúde. Secretaria de Vigilância em Saúde. Boletim Epidemiológico. Brasil Livre da Tuberculose: evolução dos cenários epidemiológicos e operacionais da doença. Brasília: Ministério da Saúde, 2019. 
. Ministério da Saúde. Secretaria de Vigilância em Saúde. Manual de Recomendações para o Controle da Tuberculose no Brasil. Brasília: Ministério da Saúde, 2018.

FERREIRA, M. R. L.; SANTOS, A. A.; ORFÃO, N. H. O vínculo no tratamento da tuberculose na atenção primária à saúde: uma revisão integrativa. Revista Brasileira em Promoção da Saúde, [s.1.], v. 32, p. 1-9, 2019.

FURLAN, M. C. R.; GONZALES, R. I. C.; MARCON, S. S. Desempenho dos serviços de controle da tuberculose em municípios do Paraná: enfoque na família. Rev. Gaúcha Enferm., Porto Alegre, v. 36, n. esp., p. 102-110, 2015.

GASPAR, L. M. da S. et al. Conhecimento, atitudes e práticas de agentes comunitários de saúde sobre tuberculose pulmonar em uma capital do Nordeste do Brasil. Ciência \& Saúde Coletiva, [s.l.], v. 24, n. 10, p. 3815-3824, out. 2019.

GEBREWELD, F. H. et al. Factors influencing adherence to tuberculosis treatment in Asmara, Eritrea: a qualitative study: a qualitative study. Journal Of Health, Population and Nutrition, [s.1.], v. 37, n. 1, p. 1-7, 5 jan. 2018.

GONZALES, R. I. C. et al. A descoberta da tuberculose no território: análise qualitativa do trabalho do agente comunitário de saúde. Cienc. enferm. Concepción, v. 21, n. 2, p. 87-97, ago. 2015 .

LINHARES, S. R. dos S. et al. A vivência do tratamento de tuberculose em unidades de Saúde da Família. Escola Anna Nery, [s.1.], v. 24, n. 2, p. 1-7, 2020.

PEREIRA, C. E. A. et al. Agentes comunitários de saúde na busca ativa do sintomático respiratório: revisão integrativa. Rev. de Enfermagem da UFPI, v. 6, n. 1, p.71-75, 2017.

PEREIRA, C. E. A. et al. O reflexo da visita domiciliar do ACS na busca ativa de um município da Amazônia. APS, Belém-PA, v. 1, n. 21, p. 77-85, jan-mar. 2018.

ROCHA, G. S. S. et al. Conhecimento dos agentes comunitários de saúde sobre a tuberculose, suas medidas de controle e tratamento diretamente observado. Cadernos de Saúde Pública, [s.1.], v. 31, n. 7, p. 1483-1496, jul. 2015.

STARFIELD, B. Atenção primária: equilíbrio entre necessidades de saúde, serviços e tecnologia. Brasília: Ministério da Saúde, 2002.

VILLA, T. C. S.; RUFFINO-NETTO, A. Tuberculose: pesquisas operacionais. 1. ed. Ribeirão Preto: FUNPEC Editora, 2009.

WORLD HEALTH ORGANIZATION. Global Tuberculosis Report 2018. Geneva: WHO, 2018. WYSOCKI, A. D. et al. Atenção Primária à Saúde e tuberculose: avaliação dos serviços: avaliação dos serviços. Revista Brasileira de Epidemiologia, [s.l.], v. 20, n. 1, p. 161-175, mar. 2017. 


\section{Abstract}

\section{Family focus about tuberculosis from the viewpoint of community health workers}

The tuberculosis (TB) control is essential the work of Community Health Agents (CHW) from diagnosis to the outcome of cases. This study aimed to analyze the dimension of family focus on TB from the perspective of CHW in Porto Velho-RO. This is a descriptive, survey-type study, carried out cross-sectionally from a quantitative approach, with the CHW who worked in PHC in the urban area of the municipality through interviews with the Primary Care Assessment TooI questionnaire (PCATool), validated for Brazil and adapted for TB care. Data were analyzed using descriptive statistics, after complying with ethical precepts. $119 \mathrm{CHW}$ were interviewed, whose perception always questioned the living conditions, knew about the people who lived with the TB patient and / or their family, requested information about the illnesses, asked about the presentation of the disease symptoms, requested sputum and / or X-ray and / or PPD and advised on the disease, treatment and other health problems. Such findings give rise to reflections on the fragility of the principles of longitudinality and integrality for greater resolution of $\mathrm{PHC}$ as an organizer of healthcare networking and coordinator of care.

Keywords: tuberculosis; community health workers; Primary Health Care; health services research. 